\title{
Stability Analysis of Switched-Linear Hybrid Systems
}

\author{
Lorenzo Sella \\ Centrum voor Wiskunde en Informatica \\ Kruislaan 413 \\ 1098 SJ Amsterdam, The Netherlands \\ Lorenzo.Sella@cwi.nl
}

\author{
Pieter Collins \\ Centrum voor Wiskunde en Informatica \\ Kruislaan 413 \\ 1098 SJ Amsterdam, The Netherlands \\ Pieter.Collins@cwi.nl
}

\begin{abstract}
In this paper we develop general techniques to study stability of hybrid systems with linear continuous dynamics. These techniques are based on matrix analysis and study of differentiable manifolds. These techniques operate on the space of switching times of the hybrid systems. Some special techniques for hybrid systems with three dimensional state space are also developed.
\end{abstract}

\section{INTRODUCTION}

A hybrid system is a mixture of continuous dynamics with discrete events system. In switched hybrid systems the discrete dynamics does not change the continuous state.

An important issue is stability, there are a number of relevant theoretical results, one of the most useful is the theorem discovered by Branicky [2]. Roughly speaking, this theorem addresses hybrid systems for which a Lyapunov function is defined for every mode. It states that the system is stable if for every Lyapunov function the sequence of values of the Lyapunov function calculated when the system enters the corresponding mode is not increasing. Other results by Hespanha and Morse [5] exploit the average dwell time, i.e. the average time the system stays in each mode. If this time is greater than a given value the system is stable. Further results by Liberzon et al. exploit Lie-algebraic conditions on the matrices to study systems with linear continuous dynamics [1], some further results can be found in [7], [6], [8], [3], [4], [9], [10].

All these results are quite theoretically important but they consist in properties that are undecidable in general (like Branicky's Theorem) or very hard to check (like average dwell time), or applicable in very few cases (like Liealgebraic conditions), so they usually do not provide an easy way to effectively decide the stability of a given general system.

In this work we study hybrid systems where each mode has linear and asymptotically stable dynamics in order to find useful results on stability for this group of systems. Even for this class the study of stability is quite complicated.

In this paper we describe a sufficient condition for stability based on the sequences of switching times corresponding to cycles of the automaton, we show how to describe this condition in a analytical and convenient way which then allow us to derive procedures based on this condition to

This work was supported by the Netherlands Organisation for Scientific Research (NWO) Vidi grant 639.032.408 and by Center for Indlejrede Software Systemer (CISS), Aalborg University, Denmark. check stability for this kind of systems. We also give a condition for instability based on the same approach.

The paper is organized as follows: we first define the framework and the problem in Section 2. In Section 3 we study cycling systems, i.e. system whose automaton is a circular graph with one directed cycle, we provide techniques to study stability. In Section 4 we address system with general switchings. In Section 5 we show special techniques for system whose state space has three dimensions. Finally in Section 6 we show an example.

\section{PROBLEM FORMULATION}

In this paper we consider the following type of hybrid system:

Definition 1: A switched linear hybrid system (s.l.h.s.) is a tuple

$$
H=(n, D, E, G)
$$

where:

1) $n$ is the dimension of the state space,

2) $D=(N, A)$ is a discrete automaton where $N$ is the set of nodes and $A \subset N \times N$ is the set of directed arcs;

3) $E=\left\{\dot{\mathbf{x}}=\mathbf{F}_{i} \mathbf{x} \mid i \in N\right\}$; is a set linear evolutions, each corresponding to same node of $\mathrm{D}$ and

4) $G=\left\{\mathbf{v}_{i j} \mathbf{x}=0 \mid(i, j) \in A\right\}$ is a set of linear guard sets, each corresponding to an arc of D. (In the intersection between two or more guard sets, $\mathbf{v}_{i j_{1}} \mathbf{x}=$ $0, \mathbf{v}_{i j_{2}} \mathbf{x}=0, \ldots$. the choice of the next mode among $j_{1}, j_{2}, \ldots$ is not deterministic)

Throughout the paper we assume that the evolution in each mode is asymptotically stable.

Definition 2: An orbit of $H$ is a triple $\left(\left\{t_{i}\right\},\left\{m_{i}\right\}, x(t)\right)$ where $t_{i}$ is the time of the $i$-th transition, $m_{i}$ is the $i$-th mode and $x(t)$ is s.t.:

1) $\dot{\mathbf{x}}(t)=\mathbf{F}_{m_{i}}(\mathbf{x}(t))$ for $t_{i}<t<t_{i+1}$

2) $\mathbf{v}_{m_{i} m_{i+1}}^{T} \mathbf{x}\left(t_{i}\right)=0$

Definition 3: The origin $\mathrm{O}$ is a globally asymptotically stable equilbrium for a switched linear hybrid system $H$ if:

1) $\forall \epsilon>0 \exists \delta>0$ such that every orbit of $H$ starting in $\mathcal{B}_{\delta}(O)$ is contained in $\mathcal{B}_{\epsilon}(O)$, and

2) every orbit of the switched linear hybrid systems converges to $\mathrm{O}$. We say a s.l.h.s. $H$ is stable if $\mathrm{O}$ is globally asymptotically stable.

An important fact is that even if each mode is stable the whole system can be unstable. The purpose of this paper 
is to find properties of this kind of systems and techniques based on these properties in order to study stability of this group of systems

Example 1: The following hybrid system, whose modes are asymptotically stable, has diverging trajectories so it is unstable:

$$
\begin{gathered}
\mathbf{F}_{1}=\left[\begin{array}{cc}
-0.1 & 1 \\
-10 & -0.1
\end{array}\right] \quad \mathbf{F}_{2}=\left[\begin{array}{cc}
-0.1 & 10 \\
-1 & -0.1
\end{array}\right] \\
\mathbf{v}_{12}=[1,0] ; \quad \mathbf{v}_{21}=[0,1]
\end{gathered}
$$

\section{CYCLIC SYSTEMS}

A key for analyzing the stability of this system is studying the periodic cycles of the hybrid automaton. So we introduce this definition:

Definition 4: Let $H=(n, D, E, G)$ be a s.l.h.s.. We say $H$ is a cyclic system with period $k$ if $\mathrm{D}$ is an oriented circular graph with only one cycle.

Trajectories switching on the cycle satisfy the following systems of equations, where $v_{k}=v_{m_{k} m_{k+1}}$ :

$$
\left\{\begin{array}{l}
\mathbf{v}_{k}^{T} \mathbf{x}=0 \\
\mathbf{v}_{1}^{T} e^{\mathbf{F}_{1} t_{1}} \mathbf{x}=0 \\
\mathbf{v}_{2}^{T} e^{\mathbf{F}_{2} t_{2}} e^{\mathbf{F}_{1} t_{1}} \mathbf{x}=0 \\
\quad \vdots \\
\mathbf{v}_{k}^{T} e^{\mathbf{F}_{n} t_{n}} \ldots e^{\mathbf{F}_{2} t_{2}} e^{\mathbf{F}_{1} t_{1}} \mathbf{x}=0 \\
\mathbf{v}_{1}^{T} e^{\mathbf{F}_{1} t_{k+1}} e^{\mathbf{F}_{n} t_{n}} \ldots e^{\mathbf{F}_{2} t_{2}} e^{\mathbf{F}_{1} t_{1}} \mathbf{x}=0 \\
\quad \vdots
\end{array}\right.
$$

Defining the $(k+1) \times n$ matrix $\mathbf{M}(\mathbf{t})$ as:

$$
\mathbf{M}(\mathbf{t})=\left[\begin{array}{c}
\mathbf{v}_{k}^{T} \\
\mathbf{v}_{1}^{T} e^{\mathbf{F}_{1} t_{1}} \\
\vdots \\
\mathbf{v}_{k}^{T} e^{\mathbf{F}_{n} t_{n}} \ldots e^{\mathbf{F}_{2} t_{2}} e^{\mathbf{F}_{1} t_{1}}
\end{array}\right]
$$

we can rewrite the equations as $\mathbf{M}(\mathbf{t}) x=0$.

In this paper the analysis of stability is developed on the study of the permanence times of the systems on each mode.

We now introduce a number of important sets which we will use throughout:

Definition 5: Consider a cycle of $k$ modes with matrix $M$. Define:

1) switching time set:

$$
\mathcal{T}=\left\{\mathbf{t}=\left[t_{1}, t_{2}, \ldots, t_{k}\right] \in \mathbb{R}^{k} \mid \operatorname{ker}(\mathbf{M}(\mathbf{t})) \neq\{0\}\right\}
$$

2) Positive switching time set:

$$
\mathcal{T}^{+}=
$$$$
\left\{\mathbf{t}=\left[t_{1}, t_{2}, \ldots, t_{k}\right] \in \mathbb{R}^{k} \mid t_{i}>0 \forall i, \operatorname{ker}(\mathbf{M}(\mathbf{t})) \neq\{0\}\right\}
$$

3) Unsafe time set:

$$
\begin{aligned}
\mathcal{U} & =\left\{\mathbf{t}=\left[t_{1}, t_{2}, \ldots, t_{k}\right] \in \mathbb{R}^{k} \mid\right. \\
t_{i} & \left.>0 \forall i,\left\|e^{F_{k} t_{k}} e^{F_{k-1} t_{k-1}} \ldots e^{F_{1} t_{1}}\right\| \geq 1\right\}
\end{aligned}
$$

4) Safe time set:

$$
\begin{aligned}
& \mathcal{S}=\left\{\mathbf{t}=\left[t_{1}, t_{2}, \ldots, t_{k}\right] \in \mathbb{R}^{k} \mid\right. \\
& \left.t_{i}>0 \forall i,\left\|e^{F_{k} t_{k}} e^{F_{k-1} t_{k-1}} \ldots e^{F_{1} t_{1}}\right\|<1\right\}
\end{aligned}
$$

Here and throughout the paper we use the euclidean norm for matrices.

\section{A. Stability analysis}

We now present some results on stability of cyclic systems.

Proposition 1: Let $H=(n, D, E, G)$ be a cyclic s.l.h.s. with Unsafe Time Set $\mathcal{U}$ and switching time manifold $\mathcal{T}$ then:

1) $\mathcal{U}$ is bounded

2) if $\mathcal{U} \neq \emptyset$ and $n=k+1$ then $\mathbf{0} \in \mathcal{T} \cap \partial \mathcal{U}$

3) if $k<n-1$, then $\mathcal{T}=\mathbb{R}^{k}$

(Proof in the appendix)

We now present a proposition from which we will develop the analysis on stability of switched linear hybrid systems:

Proposition 2: Let $H=(n, D, E, G)$ be a cyclic s.l.h.s.. If $\overline{\mathcal{U}} \cap \overline{\mathcal{T}}^{+}=\emptyset$ then the origin is globally asymptotically stable.

(Proof in the appendix)

Note that the converse is not true.

\section{B. Analytic expression of the switching time set}

We now want to get an analytic expression of the switching time set $\mathcal{T}$. We begin with the following definitions:

Definition 6: Let be:

1) $\mathbf{M}_{[i, r]}$ the matrix encompassing rows $i$-th,,.,$i+r-1$-th of $\mathbf{M}(\mathbf{t})$

2) for any $d_{1} \times d_{2}$ matrix $\mathbf{Q}(\mathbf{t})$ we define the operator cminor (consectuive minors) as follows:

- if $d_{1} \geq d_{2} \quad \operatorname{cminor}(\mathbf{Q}(\mathbf{t})) \quad=$ $\left[\operatorname{det}\left(\mathbf{Q}(\mathbf{t})_{\left.\left[1, d_{2}\right]\right)}, \ldots, \operatorname{det}\left(\mathbf{Q}(\mathbf{t})_{\left[d_{1}-d_{2}+1, d_{2}\right]}\right)\right]\right.$

- if $d_{1}<d_{2} \operatorname{cminor}(\mathbf{Q}(\mathbf{t}))=\mathbf{0}$

3) $\mathbf{L}_{S}$ a matrix obtained from $\mathbf{M}(\mathbf{t})$ removing rows contanied in $S$

4) $\hat{\mathcal{R}}_{i, r}=\left\{\left[t_{i}, \ldots, t_{i+r-1}\right] \in \mathbb{R}^{r} \mid \operatorname{rank}\left(\mathbf{M}_{[i, r]}\right)=r-1\right.$ and every subset of $r-1$ rows of $\mathbf{M}_{[i, r]}$ is linear independent $\}, r \leq n-1$

5) $\mathcal{R}_{i, r}=\left\{\mathbf{t} \in \mathbb{R}^{k} \mid\left[t_{i}, \ldots, t_{i+r-1}\right] \in \hat{\mathcal{R}}_{i, r}\right\}$

Proposition 3: Let $\mathcal{S}_{j}=\mathcal{R}_{i_{j}, r_{j}}, j: 1 \ldots z$ the finite family of all possible non empty sets among sets $\mathcal{R}_{i, r}$. Then $\mathcal{T}$ is the union of the following $2^{z}$ manifolds:

$$
\begin{gathered}
\mathcal{M}_{0}=\left\{\mathbf{t} \in \mathbb{R}^{k} \mid \operatorname{cminor}(\mathbf{M}(\mathbf{t}))=\mathbf{0} \text { and } \mathbf{t} \notin \bigcup \mathcal{S}_{j}\right\} \\
\forall N \subseteq\{1, \ldots, z\}, P(N)=\left\{r_{j} \mid j \in N\right\}: \\
\mathcal{M}_{N}=\left\{\mathbf{t} \in \mathbb{R}^{k} \mid \operatorname{cminor}\left(\mathbf{L}(\mathbf{t})_{P(N)}\right)=\mathbf{0} \text { and } \mathbf{t} \in \bigcap_{j \in N} \mathcal{S}_{j} \backslash \bigcup_{j \notin N} \mathcal{S}_{j}\right\}
\end{gathered}
$$

Let $l$ be the number of rows of $\mathbf{L}(\mathbf{t})_{P(N)}$, the dimension $d$ of the corresponding manifold is given by:

$$
d= \begin{cases}n-2 & \text { if } l \geq n-1 \\ l-1 & \text { if } l<n-1\end{cases}
$$

each point describing the evolution of a $n-d$ dimensional vector space on the initial guard set. (Proof in the appendix) 


\section{The Tangent Subspace condition}

When $n=k+1$ the origin is a crucial point for the study of condition of Proposition 2 and so stability, because Proposition 1 tells us that the boundary of the unsafe time set intersects the switching time set in the origin.

As we saw in section III-B each submanifold of the switching time set can be defined through a vector function $F(\mathbf{t})=0$, so by studying each manifold locally in the origin it is possible to find out if around the origin the unsafe time set intersects the switching time set or not. That can be done by computing the tangent subspace of the manifold in the origin, whose equation is:

$$
\left.\frac{\partial F(\mathbf{t})}{\partial \mathbf{t}}\right|_{\mathbf{t}=\mathbf{0}} \mathbf{t}=0
$$

we have the following propostion:

Proposition 4 (Tangent Subspace condition): Let $H=$ $(n, D, E, G)$ be a cyclic s.l.h.s. where $\forall i \exists t>0:\left\|e^{\mathbf{F}_{i} t}\right\|>1$ then, let $\mathcal{U}$ be the unsafe time set and $\mathcal{T}$ the switching time set. If for every manifold $\mathcal{M} \subseteq \mathcal{T}$, defined as $F(\mathbf{t})=0$, the tangent subspace does not intersect the set $\left\{\mathbf{t} \in \mathbb{R}^{n}: \mathbf{t}>\right.$ 0 or $\mathbf{t}<0\}$ - we call this condition the tangent subspace condition - then:

$$
\exists \epsilon: \mathcal{B}_{\epsilon}(\mathbf{0}) \cap \overline{\mathcal{U}} \cap \overline{\mathcal{T}}^{+}=\emptyset
$$

otherwise :

$$
\overline{\mathcal{U}} \cap \overline{\mathcal{T}}^{+} \neq \emptyset
$$

If $\exists i \forall t>0:\left\|e^{F_{i} t}\right\|>1$ then the condition still guarantees:

$$
\exists \epsilon: \mathcal{B}_{\epsilon}(\mathbf{0}) \cap \overline{\mathcal{U}} \cap \overline{\mathcal{T}}^{+}=\emptyset
$$

but if not satisfied we can not imply:

$$
\overline{\mathcal{U}} \cap \overline{\mathcal{T}}^{+} \neq \emptyset
$$

The proof is straightforward

\section{Approximation of the Unsafe Time Set by a Polyhedron}

We present now an over approximation of the unsafe time set by a polyhedron.

Definition 7: Let $H=(n, D, E, G)$ be a cyclic s.l.h.s. Define the following set:

$$
\mathcal{Y}=\left\{\mathbf{t} \in \mathbb{R}^{k} \mid \mathbf{t} \geq 0 \text { and } \mathbf{a}^{T} \mathbf{t} \geq b\right\}
$$

Here $\mathbf{a}$ and $b$ are defined as follows: $\mathbf{F}_{i}=\mathbf{T}_{i} \mathbf{J}_{i} \mathbf{T}_{i}^{-1}$, with $J_{i}$ Jordan form, let $\lambda_{i}$ the maximal real part of eigenvalues of $\mathbf{F}_{i}$. If $\lambda_{i}$ has multiplicity one in the corresponding minimal polynomial then $a_{i}=\lambda_{i}$, if the multiplicity is higher then $a_{i}=\lambda_{i}+1$,

$$
b=-\ln \left(\left\|\mathbf{T}_{1}\right\||| \mathbf{T}_{k}^{-1}\left\|\prod_{i=1}^{k-1}\right\| \mathbf{T}_{i}^{-1} \mathbf{T}_{i+1} \|\right)
$$

Proposition 5: Let $H=(n, D, E, G)$ be a cyclic s.l.h.s.. Then $\mathcal{U}$ is a subset of $\mathcal{Y}$.

Note that $\mathcal{Y}$ is unbounded if $\exists a_{i}>0$; this happens when the maximal eigenvalues of $\mathbf{F}_{i}$ has real part grater than -1 and have multiplicity higher than 1 in the minimal polynomial of the corresponding matrix.
We can now give this procedure:

Procedure 1: INPUT: $H$, OUTPUT : truth value of $\mathcal{Y} \cap$ $\overline{\mathcal{T}}^{+}=\emptyset$

1) if $n=k+1$ and the tangent subspace condition in the origin is not satisfied return false

2) compute the polyhedron overapproximation $\mathcal{Y}$ of $\mathcal{U}$

3) for every $\mathcal{M} \subseteq \mathcal{T}$ defined as $F(\mathbf{t})=0$ : compute if the origin is the unique global minimum of $\|F(\mathbf{t})\|^{2}$ in $\mathcal{Y}$ using Lagrangian multipliers, if it is not return false

4) return true

\section{E. Analytic expression of the Unsafe Time Set}

Now we want to get a convenient analytical description of $\mathcal{U}$ and $\mathcal{S}$. We use the following proposition:

Proposition 6: Given a square matrix $\mathbf{A}$ of order $n$, $\|\mathbf{A}\|<1$ iff the polynomial $p(s)=\operatorname{det}\left(\mathbf{A}-\left(\mathbf{A}^{T}\right)^{-1} s\right)$ is such that:

$$
\operatorname{sign}\left(\frac{d^{i} p}{d s^{i}}(1)\right)=\operatorname{sign}\left(\frac{d^{j} p}{d s^{j}}(1)\right)
$$

for $i, j: 0, \ldots, n$

(Proof in the appendix)

Applying this proposition to matrix $\prod_{i=1}^{k} e^{\mathbf{F}_{i} t_{i}}$ we can give the following corollary:

Corollary 1: Let $p(s)=\operatorname{det}\left(\prod_{i=1}^{k} e^{\mathbf{F}_{i} t_{i}}-\prod_{i=k}^{1} e^{-\mathbf{F}_{i} t_{i}} s\right)$ satisfies the conditions in the propostion. We can rewrite $p(s)$ as:

$$
p(s)=\sum_{i=0}^{k} p_{i}(\mathbf{t}) s^{i}
$$

where each $p_{i}(\mathbf{t})$ is written:

$$
p_{i}(\mathbf{t})=\sum_{j}^{r} q_{j}(\mathbf{t}) e^{\mathbf{I}_{j}^{T} \mathbf{t}}
$$

and $q_{j}(\mathbf{t})$ is:

- a real number if $\mathbf{F}_{1}, \ldots, \mathbf{F}_{k}$ are all diagonalizable with real eigenvalues

- a combination of functions $\sin \left(t_{i}\right), \cos \left(t_{i}\right)$ if $\mathbf{F}_{1}, \ldots, \mathbf{F}_{k}$ are diagonalizable with some complex eigenvalues

- a polynomial if $\mathbf{F}_{1}, \ldots, \mathbf{F}_{k}$ are not all diagonalizable with real eigenvalues

- a combination of polynomials and $\sin \left(t_{i}\right), \cos \left(t_{i}\right)$ in the most general case

$\mathbf{l}_{j}(\mathbf{t})$ is a function with $n$ components, each components is a polynomial function of the real parts of eigenvalues of $\mathbf{F}_{1}, \ldots, \mathbf{F}_{k}$.

$\mathcal{S}$ is described by the following systems of inequalities:

$$
\left\{\begin{array}{c}
\sum_{i=0}^{k} p_{i}(\mathbf{t})<0 \\
\sum_{i=1}^{k} i p_{i}(\mathbf{t})<0 \\
\vdots \\
\sum_{i=r}^{k} \frac{i !}{(i-r) !} p_{i}(\mathbf{t})<0 \\
\vdots \\
p_{k}(\mathbf{t})<0
\end{array}\right.
$$


Many objects are expressed as a sum of exponentials: $\sum_{j}^{r} q_{j}(\mathbf{t}) e^{\mathbf{I}_{j}^{T} \mathbf{t}}, r$ depends on the object itself. The number $r$ has to do with the complexity of dealing and analyzing the matrices and the systems they describe. So it is important to know what is precisely this number in any sum of exponential we deal with in this paper. We give the following proposition:

Proposition 7: Let $\mathbf{F}_{1} \ldots \mathbf{F}_{k}$ be square matrices of order $n$ of $H=(n, D, E, G)$ cyclic s.l.h.s. then:

1) $\sum_{j}^{r} q_{j}(\mathbf{t}) e^{\mathbf{I}_{j}^{T} \mathbf{t}}$ entry of $e^{\mathbf{F}_{i} t}: r=n$

2) $\sum_{j}^{r} q_{j}(\mathbf{t}) e^{\mathbf{I}_{j}^{T} \mathbf{t}}$ entry of $\prod_{i=1}^{k} e^{\mathbf{F}_{i} t_{i}}: r=n^{k}$

3) $\sum_{j}^{r} q_{j}(\mathbf{t}) e^{\mathbf{I}_{j}^{T} \mathbf{t}}$ entry of $\prod_{i=1}^{k} e^{\mathbf{F}_{i} t_{i}} \prod_{i=k}^{1} e^{\mathbf{F}_{i}^{T} t_{i}}: r=$ $\left(\begin{array}{c}n+1 \\ 2\end{array}\right)^{k}$

4) $\sum_{j}^{r} q_{j}(\mathbf{t}) e^{\mathbf{l}_{j}^{T} \mathbf{t}}$ term of degree $i$ of the characteristic polynomial of $\prod_{i=1}^{k} e^{\mathbf{F}_{i} t_{i}} \prod_{i=k}^{1} e^{\mathbf{F}_{i}^{T} t_{i}}: r=\left(\begin{array}{c}n+2 i-1 \\ 2 i\end{array}\right)^{k}$

5) $\sum_{j}^{r} q_{j}(\mathbf{t}) e^{\mathbf{e}_{j}^{T} \mathbf{t}}$ function defining the switching time set: $r=\prod_{i=0}^{n-1}\left(\begin{array}{c}n+i-1 \\ i\end{array}\right)$

(Proof omitted )

We can now give this procedure:

Procedure 2: INPUT: $H$, OUTPUT : truth value of $\overline{\mathcal{U}} \cap$ $\overline{\mathcal{T}}^{+}=\emptyset$

1) if $n=k+1$ and the tangent subspace condition in the origin is not satisfied return false

2) compute the analytical expression of $\mathcal{U}$

3) for every $\mathcal{M} \subseteq \mathcal{T}$ defined as $F(\mathbf{t})=0$ : compute if the origin is the unique global minimum of $\|F(\mathbf{t})\|^{2}$ in $\overline{\mathcal{U}}$ using Lagrangian multipliers, if it is not return false

4) return true

This procedure is computationally and numerically extremely more challenging than procedure 1 , because the analytical expression of $\mathcal{U}$ is in general quite complicated.

\section{F. Multiple cycling analysis}

In the previous sections we have analyzed the stability of a cycle with $k$ modes, considering the stability of one ride on the cycle, i.e. just $k$ switchings. This gives the stability condition we have described.

If we consider more the one ride we get a stronger stability condition. Let us so consider the case when the system make more than one cycle, two for instance, and define $\mathbf{P}(\mathbf{t})=$ $e^{\mathbf{F}_{n} t_{n}} \ldots e^{\mathbf{F}_{2} t_{2}} e^{\mathbf{F}_{1} t_{1}}$, we get these equations:

$$
\left\{\begin{array}{l}
\mathbf{v}_{k}^{T} \mathbf{x}=0 \\
\mathbf{v}_{1}^{T} e^{\mathbf{F}_{1} t_{1}} \mathbf{x}=0 \\
\mathbf{v}_{2}^{T} e^{\mathbf{F}_{2} t_{2}} e^{\mathbf{F}_{1} t_{1}} \mathbf{x}=0 \\
\vdots \\
\vdots \\
\mathbf{v}_{k}^{T} e^{\mathbf{F}_{n} t_{n}} \ldots e^{\mathbf{F}_{2} t_{2}} e^{\mathbf{F}_{1} t_{1}} \mathbf{x}=0 \\
\mathbf{v}_{1}^{T} \mathbf{P}(\mathbf{t}) e^{\mathbf{F}_{1} d_{1}} \mathbf{x}=0 \\
\mathbf{v}_{2}^{T} \mathbf{P}(\mathbf{t}) e^{\mathbf{F}_{2} d_{2}} e^{\mathbf{F}_{1} d_{1}} \mathbf{x}=0 \\
\vdots \\
\vdots \\
\mathbf{v}_{k}^{T} \mathbf{P}(\mathbf{t}) e^{\mathbf{F}_{n} d_{n}} \ldots e^{\mathbf{F}_{2} d_{2}} e^{\mathbf{F}_{1} d_{1}} \mathbf{x}=0
\end{array}\right.
$$

Through these equations we can define a function $h(\mathbf{t})$ : $\mathbb{R}^{k} \rightarrow \mathbb{R}^{k}$ as $h(\mathbf{t})=\mathbf{d}$
We can straightforward derive the following sufficient condition for stability and a sufficient condition for instability:

Proposition 8: The origin is globally asymptotically stable if $J_{0}=\mathcal{T} J_{i+1}=h\left(J_{i}\right) \exists \bar{i}: \forall i \geq \bar{i} J_{i} \subseteq \mathcal{S}$

Proposition 9: The origin is unstable if $\exists I_{0} \subseteq \mathcal{U} \cap \mathcal{T}$ : $I_{i+1}=h\left(I_{i}\right) \forall i I_{i} \subseteq \mathcal{U}$

\section{General SySTEMS}

So far we have just considered cycling systems. To address the study of more general systems we need to deal with a new auomaton obtained by the automaton of the system. We give this definition:

Definition 8: The evolution automaton $E A\left(N_{E A}, A_{E A}\right)$ of a s.l.h.s $H=(n, D, E, G)$ is an automaton built from $D(N, A)$ in the following way:

- $N_{E A}=A$ is the set of nodes,

- $A_{E A}=\{(a, b) \in A \times A \mid a=(i, j), b=(l, k), j=l\}$ is the set of arcs.

$(a, b)(a=(j, i)$ and $b=(i, k))$ is identified by a label $G_{i}^{j k}, i, j, k \in N$.

$G_{i}^{j k}$ is associated to the evolution described by the following equations:

$$
\left\{\begin{array}{l}
\mathbf{v}_{j i}^{T} \mathbf{x}=0 \\
\mathbf{v}_{i k}^{T} e^{\mathbf{F}_{i}(t)} \mathbf{x}=0
\end{array}\right.
$$

i.e the evolution in mode $i$ from the guard set $\mathbf{v}_{j i} \mathbf{x}=0$ to the guard set $\mathbf{v}_{i k} \mathbf{x}=0$.

Definition 9: A connecting path of $E A$ is a simple path connecting two simple cycles of $E A$ and it does not share any arc with the two cycles.

Definition 10: For a path $\pi: G_{i_{2}}^{i_{1} i_{3}}, G_{i_{3}}^{i_{2} i_{4}}, \ldots, G_{i_{r}}^{i_{r-1} i_{r+1}}$ of $E A$ we define:

$$
\mathbf{M}_{\pi}\left(t_{1}, \ldots, t_{r-1}\right)=\left[\begin{array}{c}
\mathbf{v}_{i_{1}}^{T} \\
\mathbf{v}_{i_{2}}^{T} e^{\mathbf{F}_{h\left(i_{2}\right)} t_{1}} \\
\mathbf{v}_{i_{3}}^{T} e^{\mathbf{F}_{h\left(i_{3}\right)} t_{2}} e^{\mathbf{F}_{h\left(i_{3}\right)} t_{1}} \\
\vdots \\
\mathbf{v}_{k}^{T} e^{\mathbf{F}_{h\left(i_{r}\right)} t_{r-1}} \ldots e^{\mathbf{F}_{h\left(i_{3}\right)} t_{2}} e^{\mathbf{F}_{h\left(i_{1}\right)} t_{1}}
\end{array}\right]
$$

Positive switching time set:

$$
\begin{aligned}
& \mathcal{T}_{\pi}^{+}= \\
& \left\{\mathbf{t}=\left[t_{1}, t_{2}, \ldots, t_{r-1}\right] \in \mathbb{R}^{r-1} t_{i}>0 \mid \operatorname{ker}\left(\mathbf{M}_{\pi}(\mathbf{t})\right) \neq\{0\}\right\} \\
& \quad \text { Unsafe time set: } \\
& \mathcal{U}_{\pi}=\left\{\mathbf{t}=\left[t_{1}, t_{2}, \ldots, t_{r-1}\right] \in \mathbb{R}^{r-1} \mid\right. \\
& \left.t_{i}>0 \forall i,\left\|e^{F_{h\left(i_{r}\right)} t_{r-1}} e^{F_{h\left(i_{r-1}\right)} t_{r-1}} \ldots e^{F_{1} t_{1}}\right\| \geq 1\right\} \\
& \text { we say } \pi \text { stable if: }
\end{aligned}
$$

$$
\overline{\mathcal{U}_{\pi}} \cap \overline{\mathcal{T}}^{+}=\emptyset
$$

Proposition 10: The hybrid $H=(n, D, E, G)$ system is asymptotically stable if every simple cycle and every connecting path $\gamma$ satisfies the condition $\overline{\mathcal{U}_{\gamma}} \cap \overline{\mathcal{T}}_{\gamma}{ }^{+}=\emptyset$.

Proof: We just notice that every trajectory with infinitely many switchings corresponds to an infinite sequence of simple cycles and connecting paths. The above condition implies that every simple cycle and every connecting path 
is norm contracting and we directly prove stability of the system like in the proof of Proposition 2.

Example 2: In Fig. 1 we show an automaton and in Fig. 2 we show the evolution automaton. The simple cycles are :

$$
G_{1}^{22} G_{2}^{11} G_{1}^{33} G_{3}^{11} G_{1}^{23} G_{3}^{12} G_{2}^{31} G_{1}^{23} G_{3}^{11} G_{1}^{32} G_{2}^{11}
$$

the connecting paths are:

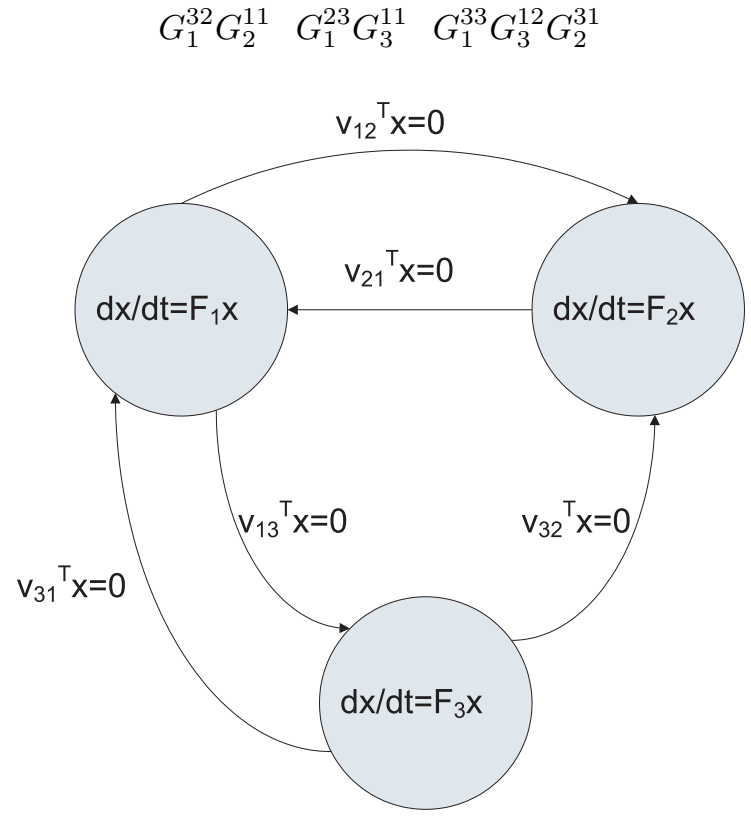

Fig. 1.

\section{CYCLIC SWITCHING IN TWO AND THREE DIMENSIONS}

\section{A. Cyclic switching in two dimensions}

Let us consider a mode where $\mathbf{v}_{0}^{T} \mathbf{x}=0$ is the equation of the in-guard set, $\mathbf{v}_{1}^{T} \mathbf{x}=0$ is the equation of its out-guard set. We have the following equations:

$$
\left\{\begin{array}{l}
\mathbf{v}_{0}^{T} \mathbf{x}=0 \\
\mathbf{v}_{1}^{T} e^{F t} \mathbf{x}=0
\end{array}\right.
$$

$\mathbf{M}(t) \mathbf{x}=0 t>0: \operatorname{det}(\mathbf{M}(t))=0$ is the permanence time of the system in the mode. $\left\|e^{\mathbf{F} t}\right\|$ is the gain of the node.

Proposition 11: The origin is a asymptotically stable if and only if the gain of every cycle is lower than one.

\section{B. Cyclic switching in three dimensions}

Let us consider the cycling hybrid system in figure 3 .

The number of modes $k$ is two, the equations of the switching sets are the hyperplanes $\mathbf{v}_{1}^{T} \mathbf{x}=0$ and $\mathbf{v}_{2}^{T} \mathbf{x}=0$. We can write the following system of equations:

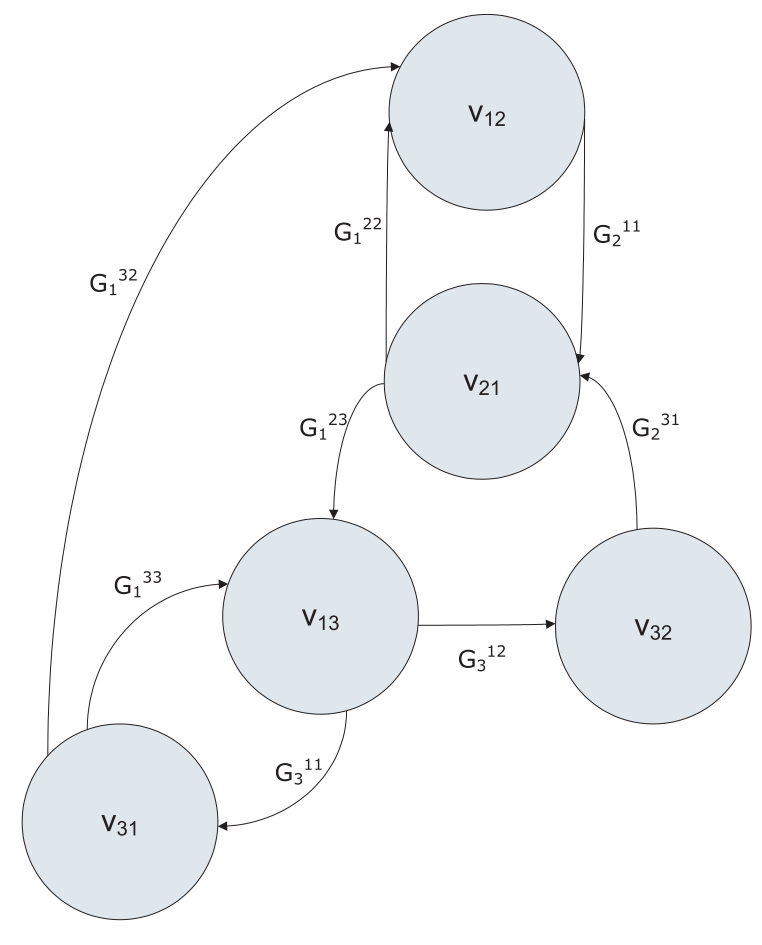

Fig. 2.

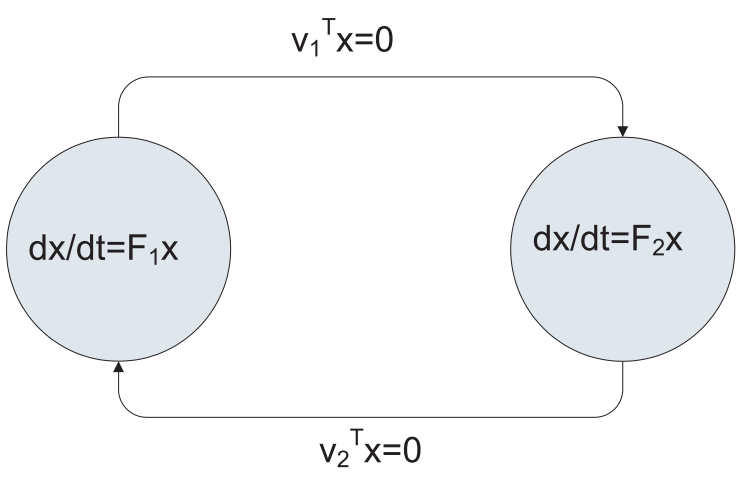

Fig. 3.

$$
\left\{\begin{array}{l}
\mathbf{v}_{2}^{T} \mathbf{x}=0 \\
\mathbf{v}_{1}^{T} e^{\mathbf{F}_{1} t_{1}} \mathbf{x}=0 \\
\mathbf{v}_{2}^{T} e^{\mathbf{F}_{2} t_{2}} e^{\mathbf{F}_{1} t_{1}} \mathbf{x}=0
\end{array}\right.
$$

In this case the matrix associated to the system is:

$$
\begin{gathered}
\mathbf{M}=\left[\begin{array}{c}
\mathbf{v}_{2}^{T} \\
\mathbf{v}_{1}^{T} e^{\mathbf{F}_{1} t_{1}} \\
\mathbf{v}_{2}^{T} e^{\mathbf{F}_{2} t_{2}} e^{\mathbf{F}_{1} t_{1}}
\end{array}\right] \\
f\left(t_{1}, t_{2}\right)=\operatorname{det}(\mathbf{M})=\sum_{i, j} p_{i j}\left(t_{1}, t_{2}\right) e^{\mu_{i} t_{2}+\lambda_{j} t_{1}}
\end{gathered}
$$

with $\lambda_{i}, \mu_{i}$ eigenvalues of $\mathbf{F}_{1}, \mathbf{F}_{2}$ respectively for $i: 1 \ldots n$ , $p_{i j}\left(t_{1}, t_{2}\right)$ are polynomials, trigonometric functions or a combination of both. 
Proposition 12: It is possible to compute the number of solutions of the equation $f\left(t_{1}, t_{2}\right)=0$ in the unknown variable $t_{1}$ just by looking at sings of some exponential functions of $t_{2}$ and parameters of $f\left(t_{1}, t_{2}\right)$ (the same hold if we consider $t_{2}$ as unknown variable).

\section{Stability condition}

It is possible to check the conditions of Proposition 2 by using Procedure 2, but in this case we can provide a simpler procedure in which we do not need Lagrangian multipliers techniques. Let us describe this procedure for the intersection $\mathcal{W} \cap \overline{\mathcal{T}}^{+}=\emptyset$, where $\mathcal{W}$ can be either $\overline{\mathcal{U}}$ or $\mathcal{Y}$.

Definition 11: Let $\bar{t}_{1}$ be the rightmost point of $\mathcal{Y}$ on $t_{1}$ axis, then according Proposition 12 on the interval $\left[0, \bar{t}_{1}\right]$ we can define a computable piecewise constant function:

$$
s\left(t_{1}\right):\left[0, \bar{t}_{1}\right] \longrightarrow \mathbb{N} \cup\{\infty\}
$$

which gives the number of points of the $\mathcal{T}$ at coordinate $t_{1}$. According that we can understand the structure of $\mathcal{T}$ in the region.

- If $F_{1}$ has only real eigenvalues, the function is either constant with value 1 or 2 or piecewise constant with value 0 and 2 .

- If $F_{1}$ has a couple of complex conjugate eigenvalues, the function can be piecewise constant with arbitrary non negative value, even infinite.

Procedure 3: INPUT: $H$, OUTPUT: truth value of $\overline{\mathcal{W}} \cap$ $\overline{\mathcal{T}}^{+}=\emptyset(\mathcal{W}$ can be either $\overline{\mathcal{U}}$ or $\mathcal{Y})$.

1) if the tangent hyperplane condition is not satisfied return false

2) if $\partial \mathcal{W} \cap \mathcal{T} \neq\{0\}$ return false ( $\partial \mathcal{W} \cap \mathcal{T}$ is computed by solving a system of nonlinear equations, the ones defining $\partial \mathcal{W}$ and $\mathcal{T}$ )

3) compute $\bar{t}_{1}$, i.e. the rightmost point of $\mathcal{Y}$ on $t_{1}$-axis

4) compute $s\left(t_{1}\right)$

5) partition $\left[0, \bar{t}_{1}\right]$ correspondingly to the constant pieces of $s:\left[\tau_{0}=0, \tau_{1}\right],\left[\tau_{1}, \tau_{2}\right] \ldots\left[\tau_{r-1}, \tau_{r}\right]$

6) for each $\left[\tau_{i}, \tau_{i+1}\right]$ such that $\left.s(t)>0 \forall t \in\right] \tau_{i}, \tau_{i+1}[$, choose arbitrarily a point $\tilde{t} \in] \tau_{i}, \tau_{i+1}[$, compute the set $\mathcal{M}_{i}=\left\{\left(t_{1}, t_{2}\right) \in \mathcal{W} \cap \overline{\mathcal{T}}^{+} \mid t_{2}=\tilde{t}\right\}=\left\{\left(\tilde{t}, t_{2}\right) \mid\right.$ $\operatorname{det}\left(M\left(\tilde{t}, t_{2}\right)\right)=0$ and $\left.\left(\tilde{t}, t_{2}\right) \in \mathcal{W}\right\}$

7) if $\bigcup_{i} \mathcal{M}_{i}=\emptyset$ return true, else return false

Considerations:

- if $s$ is constant $\mathcal{T}$ has a number of branches, one intersect $\partial \mathcal{W}$ only in the origin but does not intersect the interior of $\mathcal{W}$, the others do not intersect $\partial \mathcal{W}$ at all, so by continuity of $\mathcal{T}$ they do not intersect the interior of $\mathcal{W}$, and so $\mathcal{W} \cap \overline{\mathcal{T}}^{+}=\emptyset$ (at least if there are no singularities)

- if $s$ is not constant this means that there are some closed curves for which we can easily test the membership.

- if $\partial \mathcal{W} \cap \mathcal{T}=\{0\}$ this means that $\mathcal{W} \cap \overline{\mathcal{T}}^{+}$could be either an empty set or a number of closed curves

- $\left|\mathcal{M}_{i}\right| \leq s(\tilde{t})$
Note if there are not complex eigenvalues the structure of $\mathcal{T}$ and the procedure is much simpler because the upper bound for $s$ is 2 , so if only one matrix has complex eigenvalues we should apply the procedure using the other one.

1) Case $k>2$ :

In the case the number of modes is greater than two $\forall i, r$ $\hat{\mathcal{R}}_{i, r}=\emptyset$, then $\mathcal{T}=\mathcal{M}_{0}$ :

Let $f_{i}\left(t_{i}, t_{i+1}\right)=(\operatorname{cminor}(\mathbf{M}(\mathbf{t})))_{i}$ :

$$
\mathcal{M}_{0}=\left\{\begin{array}{l}
f_{1}\left(t_{1}, t_{2}\right)=0 \\
f_{2}\left(t_{2}, t_{3}\right)=0 \\
\vdots \\
f_{k-1}\left(t_{k-1}, t_{k}\right)=0
\end{array}\right.
$$

Definition 12: We define:

$$
\begin{gathered}
s_{i}\left(t_{i}\right)=\left|\left\{t_{i+1} \mid f_{i}\left(t_{i}, t_{i+1}\right)=0\right\}\right| \\
c_{i}\left(t_{i+1}\right)=\left|\left\{t_{i} \mid f_{i}\left(t_{i}, t_{i+1}\right)=0\right\}\right| \\
\hat{s}_{1}\left(t_{1}\right)=s_{1}\left(t_{1}\right) \hat{c}_{1}\left(t_{2}\right)=c_{1}\left(t_{2}\right) \\
\hat{s}_{i}\left(t_{i}\right)=\left|\left\{t_{i+1} \mid f\left(t_{i}, t_{i+1}\right)=0, \hat{c}_{i-1}\left(t_{i}\right) \neq 0\right\}\right| \\
\hat{c}_{i}\left(t_{i+1}\right)=\left|\left\{t_{i} \mid f\left(t_{i}, t_{i+1}\right)=0, \hat{s}_{i}\left(t_{i}\right) \neq 0\right\}\right|
\end{gathered}
$$

We can compute the above piecewise constant functions exploiting Proposition 12. We give the following procedure:

Procedure 4: INPUT: $H$, OUTPUT: truth value of $\overline{\mathcal{W}} \cap$ $\overline{\mathcal{T}}^{+}=\emptyset(\mathcal{W}$ can be either $\overline{\mathcal{U}}$ or $\mathcal{Y})$.

1) if $\partial \mathcal{W} \cap \mathcal{T} \neq\{0\}$ return false ( $\partial \mathcal{W} \cap \mathcal{T}$ is computed by solving a system of nonlinear equations, the ones defining $\partial \mathcal{W}$ and $\mathcal{T}$ )

2) compute $\bar{t}_{k}=\max _{t_{k}}\left\{t_{k} \mid\left[t_{1}, \ldots, t_{k}\right] \in \mathcal{Y}\right\}$.

3) compute $\hat{c}_{k-1}\left(t_{k}\right)$

4) partition $\left[0, \bar{t}_{k}\right]$ correspondingly to the constant pieces of $\hat{c}_{k-1}\left(t_{k}\right):\left[\tau_{0}=0, \tau_{1}\right],\left[\tau_{1}, \tau_{2}\right] \ldots\left[\tau_{r-1}, \tau_{r}\right]$

5) for each $\left[\tau_{i}, \tau_{i+1}\right]$ such that $\left.s(t)>0 \forall t \in\right] \tau_{i}, \tau_{i+1}[$, choose arbitrarily a point $\tilde{t} \in] \tau_{i}, \tau_{i+1}[$, compute the set $\mathcal{M}_{i}=\left\{\left(t_{1}, \ldots, t_{k}\right) \in \mathcal{W} \cap \overline{\mathcal{T}}^{+} \mid t_{k}=\tilde{t}\right\}$

6) if $\bigcup_{i} \mathcal{M}_{i}=\emptyset$ return true else return false

\section{EXAMPLE}

Let us consider the s.l.h.s. system defined by the following matrices and vectors, and the automaton in Fig. 5:

$$
\begin{aligned}
& \mathbf{F}_{1}=\left[\begin{array}{ccc}
-1 & -1 & 0 \\
2 & -4 & 1 \\
2 & -2 & 0
\end{array}\right], \mathbf{F}_{2}=\left[\begin{array}{lll}
-7 & 4 & 6 \\
-3 & 1 & 3 \\
-3 & 2 & 2
\end{array}\right], \mathbf{F}_{3}=\left[\begin{array}{ccc}
1 & 1 & -2 \\
-10 & -6 & 6 \\
-2 & -1 & -1
\end{array}\right] \\
& \mathbf{v}_{12}=\left[\begin{array}{c}
1 \\
0 \\
-1
\end{array}\right], \mathbf{v}_{21}=\left[\begin{array}{c}
1 \\
-3 \\
2
\end{array}\right], \mathbf{v}_{13}=\left[\begin{array}{c}
-11 \\
3 \\
-12
\end{array}\right], \mathbf{v}_{32}=\left[\begin{array}{c}
8 \\
-6 \\
10
\end{array}\right]
\end{aligned}
$$

The derived automaton is shown im in Fig. 5 (second automaton). To check stability we have to check stability of simple cycles: $G_{1}^{22} G_{2}^{11}$ and $G_{1}^{23} G_{3}^{12} G_{2}^{31}$. 


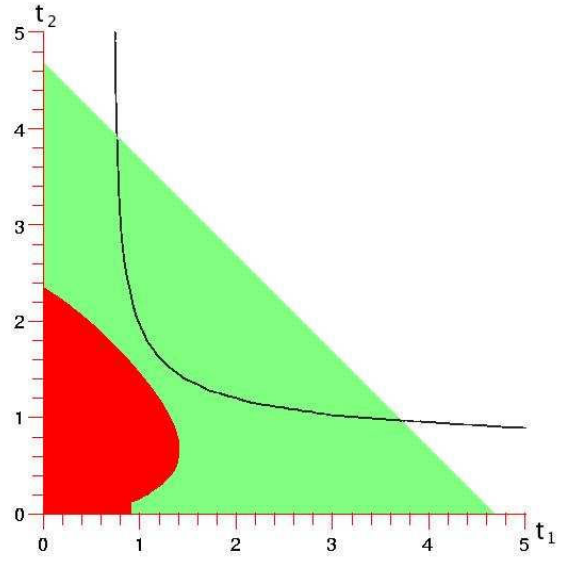

Fig. 4.

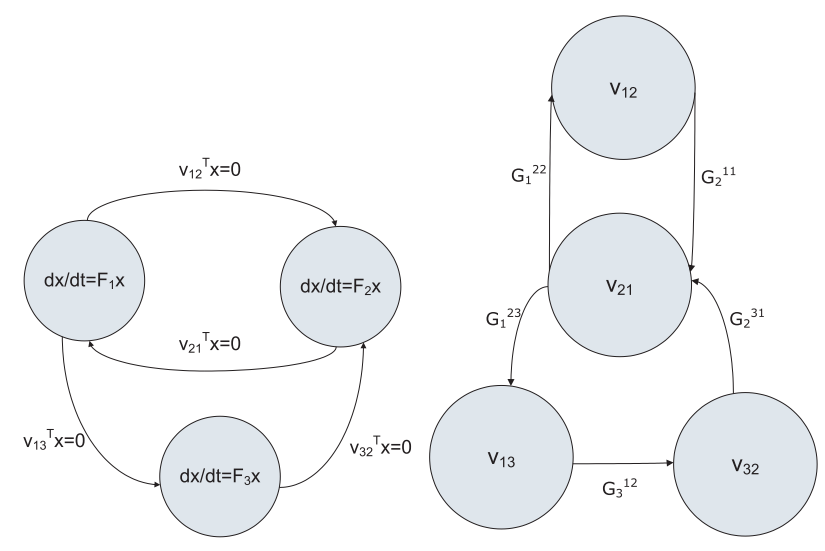

Fig. 5.

First cycle $G_{1}^{22} G_{2}^{11}$ : we apply Procedure 3 , the analytical expression of $\mathcal{T}$ is :

$3 e^{t_{1}-t_{2}}-12 e^{t_{1}-2 t_{2}}+\left(3-6 t_{1}\right) e^{2 t_{1}-t_{2}}+\left(6+12 t_{1}\right) e^{2 t_{1}-2 t_{2}}=0$

The tangent subspace at the origin is: $15 t_{1}+6 t_{2}=0$ which does not intersect the region $\left\{\left(t_{1}, t_{2}\right) \mid t_{1}>0, t_{2}>0\right\}$, so the tangent subspace condition is satisfied. We have:

$$
\mathcal{Y}=\left\{\left(t_{1}, t_{2}\right): t_{1}, t_{2} \geq 0,-t_{1}-t_{2} \geq b\right\}
$$

where $b=-\ln \left(\left\|T_{1}|||| T_{1}^{-1} T_{2}\right\|\left\|T_{2}^{-1}\right\|\right) \approx-4.6734$. In this case $\mathcal{Y} \cap \mathcal{T} \neq \emptyset$ as shown in Fig. 4, where the black line is $\mathcal{T}$ and the green shaded triangle $\mathcal{Y}$. The unsafe region is described analytically by three inequalities each having about 20 terms. We computed that $\partial \mathcal{U} \cap \mathcal{T}^{+}=\emptyset$

$$
s\left(t_{1}\right)=\left\{\begin{array}{l}
0 \text { for } 0<t_{1}<q \\
1 \text { for } q<t<\bar{t}_{1}
\end{array}\right.
$$

where $\bar{t}_{1}=-b, q \approx 0.7388$.

Let us choose a point in the open interval $\left(q, \bar{t}_{1}\right): \hat{t}_{1}=2$, $\hat{t}_{2} \approx 1.1945$ such that $\left(\hat{t}_{1}, \hat{t}_{2}\right) \in \mathcal{T}$. $\left\|e^{\mathbf{F}_{1} \hat{t}_{1}} e^{\mathbf{F}_{2} \hat{t}_{2}}\right\| \approx 0.4948$, the cycle is stable, as it shows in Fig.4, where $\mathcal{U}$ is the red region.
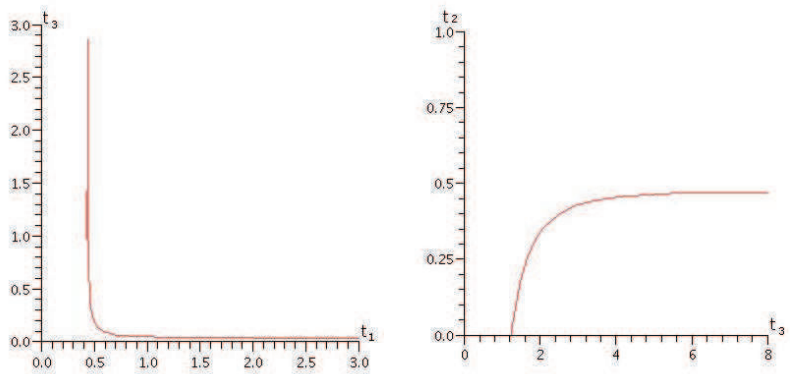

Fig. 6.

Second cycle $G_{1}^{23} G_{3}^{12} G_{2}^{31}$, we apply Procedure 4, we have:

$$
\mathcal{Y}=\left\{\left(t_{1}, t_{2}\right): t_{1}, t_{2} \geq 0,-t_{1}-t_{2}-t_{3} \geq b\right\}
$$

where $b \approx-8.4574$. We compute that $\partial \mathcal{U} \cap \mathcal{T}^{+}=\emptyset$. The projections of the manifold on the plane $t_{1}, t_{3}$ and $t_{3}, t_{2}$ are shown in figure 6 . We can see that:

$\hat{s}\left(t_{1}\right)=\left\{\begin{array}{l}0 \text { for } 0<t_{1}<q_{1} \\ 1 \text { for } q<t<\bar{t}_{1}\end{array} \quad \hat{s}\left(t_{3}\right)=\left\{\begin{array}{l}0 \text { for } 0<t_{1}<q_{2} \\ 1 \text { for } q<t<\bar{t}_{3}\end{array}\right.\right.$

where $\bar{t}_{1}=\bar{t}_{3}=-b, q_{1} \approx 0.4332, q_{2} \approx 1.6011$. Let us choose a point $\hat{t}=\left(\hat{t}_{1} \approx 0.4348, \hat{t}_{2} \approx 0.2460, \hat{t}_{3}=2\right)$, $\left\|e^{\mathbf{F}_{1} \hat{t}_{1}} e^{\mathbf{F}_{3} \hat{t}_{3}} e^{\mathbf{F}_{2} \hat{t}_{2}}\right\| \approx 0.2816 . \mathcal{M}_{1}=\emptyset$. The cycle is stable. Since every cycle of the system a combination of these two cycles the system is stable by Proposition 10 .

\section{CONCLUSION AND FUture WORK}

In this paper we have analyzed stability of switched linear hybrid systems. We have developed techniques to check stability for system of any dimension and any automaton topology. We have shown special techniques for three dimensional systems.

New techniques exploiting Lyapunov functions and a stabilizability approach using these results are currently being developed.

\section{APPENDIX}

Proof: [Proposition1]

1) Because of asymptotically stability of each mode, the equality

$$
\lim _{\mathbf{t} \rightarrow \infty}\left\|e^{\mathbf{F}_{k} t_{k}} e^{\mathbf{F}_{k-1} t_{k-1}} \ldots e^{\mathbf{F}_{1} t_{1}}\right\|=0
$$

is true in any positive direction of $\mathbf{t}=\left[t_{1}, t_{2}, \ldots, t_{k}\right]$, so $\mathcal{U}$ is bounded.

2) By definition of $\mathcal{U}, \mathbf{0} \notin \mathcal{U}$, but if $\mathcal{U} \neq \emptyset$ then $\mathbf{0} \in \partial \mathcal{U}$ because $\left\|e^{\mathbf{F}_{k} 0} e^{\mathbf{F}_{k-1} 0} \ldots e^{\mathbf{F}_{1} 0}\right\|=\|I I \ldots I\|=1$ and $\mathbf{0} \in \mathcal{T}$ since $\operatorname{rank}(\mathbf{M}(\mathbf{0}))<n$.

3) $\forall \mathbf{t} \in \mathbb{R}^{k} \operatorname{rank}(\mathbf{M}(\mathbf{t})) \leq k+1<n$, so $\operatorname{ker}(\mathbf{M}(\mathbf{t})) \neq\{0\}$ and hence $\mathbf{t} \in \mathcal{T}$.

Proof: [Proposition 2] We consider only trajectories with infinity many switchings because since the modes are asymptotically stable, asymptotically stability of the origin in the case of finite many switchings is already ensured.

After $m \times k$ switchings every trajectory satisfies the equivalence:

$$
\left.x_{m}^{T}=x^{T} \quad \sum_{i=0}^{m-1} \sum_{l=1}^{k} t_{i k+l}\right)=x_{0}^{T} \prod_{i=0}^{m-1} \prod_{l=1}^{k} e^{\mathbf{F}_{l}^{T} t_{i k+l}}
$$


If $\overline{\mathcal{U}} \cap \overline{\mathcal{T}}^{+}=\emptyset$ then $\left\|\prod_{l=1}^{k} e^{\mathbf{F}_{l}^{T} t_{i k+l}}\right\|<1 \forall i$, so $\left\|x_{p}\right\| \leq\left\|x_{q}\right\| \forall p \geq q$, so the origin is globally stable.

Since $\overline{\mathcal{U}}$ is compact and $\overline{\mathcal{T}}^{+}$is closed the distance between them is greater then zero, so $\exists \epsilon$ : $\left\|\prod_{l=1}^{k} e^{\mathbf{F}_{l}^{T} t_{i k+l}}\right\|<1-\epsilon \forall i$ then

$$
\lim _{k \rightarrow \infty}\left\|\prod_{i=0}^{m-1} \prod_{l=1}^{k} e^{\mathbf{F}_{l}^{T} t_{i k+l}}\right\| \leq \lim _{k \rightarrow \infty} \prod_{i=0}^{m-1}\left\|\prod_{l=1}^{k} e^{\mathbf{F}_{l}^{T} t_{i k+l}}\right\|=0
$$

so the equilibrium is globally asymptotically stable.

Proof: [Proposition 3] First of all we prove that $\hat{\mathcal{R}}_{i, r}$ is a manifold. To do that we just need to notice that we can describe the following condition: $\operatorname{rank}\left(\boldsymbol{M}_{[i, r]}\right)=r-1$ and every set of $r-1$ rows of $\boldsymbol{M}_{[i, r]}$ is linear independent, by a number of constraints obtained by Gaussian elimination, more precisely applying the pivoting Gaussian elimination on $\mathbf{M}_{[i, r]}$ and then imposing the entries of the last row equal to zero and imposing at least one element of the second last row different from zero.

$\cup \mathcal{M}_{N} \cup \mathcal{M}_{0} \subseteq \mathcal{T}:$

- $\forall \mathbf{t} \in \mathcal{M}_{0}$ the rows of $\mathbf{M}(\mathbf{t})$ are a linear combinations of the first $n-1$ rows of $\mathbf{M}(\mathbf{t})$, so $\mathbf{M}(\mathbf{t})$ does not have full rank.

- $\forall \mathbf{t} \in \mathcal{M}_{N}$ the rows of $\mathbf{M}(\mathbf{t})$ are a linear combinations of the rows of $\mathbf{L}(\mathbf{t})_{P(N)}$ if this matrix has no more the $n-1$ rows, otherwise they are linear combinations of the first $n-1$ rows of $\mathbf{L}(\mathbf{t})_{P(N)}$, so $\mathbf{M}(\mathbf{t})$ does not have full rank.

In either case, $\operatorname{ker}(\mathbf{M}(\mathbf{t})) \neq\{0\}$.

$\mathcal{T} \subseteq \cup \mathcal{M}_{N} \cup \mathcal{M}_{0}:$

Consider $\overline{\mathbf{t}} \in \mathcal{T}$, this implies that $\operatorname{cminor}(\mathbf{M}(\overline{\mathbf{t}}))=\mathbf{0}$ and $\left.\operatorname{cminor}(\mathbf{L}(\overline{\mathbf{t}}))_{P(N)}\right)=\mathbf{0} \forall N$.

We assume that $\overline{\mathbf{t}}$ does not belong to any manifold. So beacuse $\overline{\mathbf{t}} \notin \mathcal{M}_{0}$ then $\overline{\mathbf{t}} \in \bigcup \mathcal{S}_{j}$. So $\exists N$ such that $\overline{\mathbf{t}} \in \bigcap_{j \in N} \mathcal{S}_{j}$ but beacuse $\overline{\mathbf{t}} \notin \mathcal{M}_{N}$ then $\overline{\mathbf{t}} \notin \bigcap_{j \in N} \mathcal{S}_{j} \backslash \bigcup_{j \notin N} \mathcal{S}_{j}$, so this means $\overline{\mathbf{t}} \in \mathcal{S}_{j}$ for some $j \notin N$. Repeating this step proves $\overline{\mathbf{t}} \in \bigcap \mathcal{S}_{j}$ which means $\overline{\mathbf{t}} \in \mathcal{M}_{\{1, \ldots, z\}}$, a contradiction.

Finally we notice that:

- $\mathcal{M}_{0}$ is defined by constraints in $\operatorname{cminor}(\mathbf{M}(\mathbf{t}))=\mathbf{0}$ which are $k+$ $1-(n-1)$ in a space of $k$ varaibles, so the dimension of $\mathcal{M}_{0}$ is $k-[k+1-(n-1)]=n-2$. And because $\mathcal{M}_{0}$ has rank $n-1$ each point describes the evolution of one dimensional vector space in the initial guard set.

- $\mathcal{M}_{N}$ is defined by constraints in $\operatorname{cminor}\left(\mathbf{L}(\mathbf{t})_{P(N)}\right)=\mathbf{0}$ and $\bigcap_{j \in N} \mathcal{S}_{j} \backslash \bigcup_{j \notin N} \mathcal{S}_{j}:$

- if $l \geq n-1$ the first constraints are $l-(n-1)$ and the second ones are $k+1-l$, so in total the constraints are still $k+1-(n-1)$ the dimension of $\mathcal{M}_{N}$ is $k-[k+1-(n-1)]=n-2$. Each point describes the evolution of one dimensional vector space on the initial guard set.

- if $l<n-1$ the first constraints are zero and the second ones are $k+1-l$, the dimension of $\mathcal{M}_{N}$ is $l-1$. Each point describes the evolution of $n-l$ dimensional vector space on the initial guard set because $\mathbf{M}(\mathbf{t})$ has rank $l$.

Proof: [Proposition 5] The norm of a block-diagonal matrix is the maximal norm among the norms of its blocks. If $\mathbf{J}$ is a Jordan matrix then $e^{\mathbf{J} t}$ is block-diagonal matrix. The multiplicity of an eigenvalue in the minimal polynomial is the dimension of his bigger block in the Jordan form. If a block $\mathbf{B}$ has dimension one then $\|\mathbf{B}\|=e^{\operatorname{Re}(\mu) t}$ where $\mu$ is the corresponding eigenvalue. If the block $\mathbf{B}$ has dimension $k>1$, let us write $\mathbf{B}=e^{\mu t} \mathbf{C}$, then because $\|\mathbf{C}\|^{2} \leq\|\mathbf{C}\|_{1}\|\mathbf{C}\|_{\infty}$ and in this case $\|\mathbf{C}\|_{1}=\|\mathbf{C}\|_{\infty}=\sum_{i=0}^{k} \frac{t^{k}}{k !} \leq e^{t}$, we have $\|\mathbf{B}\| \leq e^{(R e(\mu)+1) t}$. Finally:

$\left\|\prod_{i=1}^{k} e^{\mathbf{F}_{i} t_{i}}\right\| \leq \prod_{i=1}^{k}\left\|e^{\mathbf{F}_{i} t_{i}}\right\|=\prod_{i=1}^{k}\left\|e^{\mathbf{T}_{i} \mathbf{J}_{1} \mathbf{T}_{i}^{-1} t_{i}}\right\|=$ $\prod_{i=1}^{k}\left\|\mathbf{T}_{i} e^{\mathbf{J}_{i} t_{i}} \mathbf{T}_{i}^{-1}\right\| \leq$

$\prod_{i=1}^{k}\left\|e^{\mathbf{J}_{i} t_{i}}\right\|\left\|\mathbf{T}_{1}\right\| \prod_{i=1}^{k-1}\left\|\mathbf{T}_{i}^{-1} \mathbf{T}_{i+1}\right\|\left\|\mid \mathbf{T}_{k}^{-1}\right\|$

$e^{\mathbf{a}^{T} \mathbf{t}}\left\|T_{1}\right\| \prod_{i=1}^{k-1}\left\|\mathbf{T}_{i}^{-1} \mathbf{T}_{i+1}\right\|\left\|\mid \mathbf{T}_{k}^{-1}\right\|$

Proposition 13: Given a polynomial $p(s)$ whose zeros are all real, all zeroes of $p^{(1)}(s)$ are real and they all belonged to the interval $\left[s_{1}, s_{n}\right]$, where $s_{1}$ and $s_{n}$ are respectively the lowest and the greatest zeros of $p(s)$. Proof: Let first assume $p(s)$ has $n$ different zeroes, then it must have $n-1$ different stationary points, which are the zeroes of $p^{(1)}(s)$ and of course they all belonged to the interval $\left[s_{1}, s_{n}\right]$. If $b$ has multaplicity $k>1$, it is possible to write $p(s)=(s-b)^{k} q(s), p^{(1)}(s)=k(s-b)^{k-1} q(s)+(s-$ $b)^{k} q^{(1)}(s)$, so $b$ has multplicity $k-1$ in $p^{(1)}(s)$. So the resutls still holds.

Proof: [Proposition 6] First of all we notice that the zeroes of $\mathrm{p}(\mathrm{s})$ are the squares of the singular values of $A$, being $p(s)=\operatorname{det}\left(A A^{T}-\right.$ $I s) \operatorname{det}\left(\left(A^{T}\right)^{-1}\right)$, which means that every zeros of $p(s)$ is real and positive. By definition the greatest zero of $p(s)$ is the square of $\|A\|$ so $\|A\|<1$ iff every zero of $p(s)$ belongs to the interval $[0,1)$

Let assume every zero of $p(s)$ belongs to the interval $[0,1)$, for propostion 13 the zeroes of any derivative of $p(s)$ belong to that interval $[0,1)$, so every function $p(s)^{(k)} k: 0, \ldots, n-1$ has constant sign in $[1-\epsilon,+\infty)$. Beacuse $\lim _{s \rightarrow+\infty} p(s)=\lim _{s \rightarrow+\infty} p(s)^{(1)}$ (when $\mathrm{p}(\mathrm{s})$ has degree higher than 2 ) the sign of all these functions in $[1-\epsilon,+\infty)$ is the same.

$$
\Leftarrow
$$

Let us write $\frac{d^{j} p}{d s^{j}}(s)$ as $p^{(j)}(s)$. Let assume $\operatorname{sign}\left(p^{(k)}(1)\right)=$ $\operatorname{sign}\left(p^{(j)}(1)\right)$ for $j, k: 0, \ldots, n$, by hypothesis we know that $p(s)$ has real postive zeroes and so for proposition $13 p(s)^{(k)} k: 0, \ldots, n-1$ have real positive zeroes, let use induction:

- base step: let assume $p(s)$ has degree one, if $\operatorname{sign}(p(1))=$ $\operatorname{sign}\left(p^{(1)}\right)$,then $p(s)$ has constant sign in $[1,+\infty)$ and so his zero, being positive, belongs to $[0,1)$

- inductive step: by inductive hypothesis every zero of $p^{(1)}(s)$ belongs to $[0,1)$, in $[1,+\infty) p^{(1)}(s)$ has constant sign, so $p(s)$ is monotonic in such interval, besides $\operatorname{sign}(p(+\infty))=\operatorname{sign}\left(p^{(1)}(+\infty)\right)=$ $\operatorname{sign}\left(p^{(1)}(1)\right)=\operatorname{sign}(p(1))$ then also $p(s)$ has constant sign in $[1,+\infty)$ so every zero of $p(s)$ belongs to $[0,1)$.

\section{REFERENCES}

[1] A. S. Morse A. Liberzon, J.P. Hespanha. Stability of switched systems: a Lie-algebraic condition. Technical report, Department of Electrical Engineering, Yale University, 1999.

[2] M.S. Branicky. Multiple Lyapunov functions and other analysis tools for switched and hybrid systems. IEEE Transactions on automatic control, 43(4), April 1998.

[3] R. A Decarlo, M. S. Branicky, S. Petterson, and B.Lennartson. Perspective and results on the stability and stabizability of hybrid systems. Proceedings of the IEEE, 88(7), July 2000.

[4] J.P. Hespanha. Extending LaSalle's invariance principle to switched linear systems. In Proceedings of the 40-th IEEE Conference on Decision and Control, 2001.

[5] J.P. Hespanha and A. S. Morse. Stability of switched systems with average dwell-time. In Proceedings of 38-th IEEE Conference on Decision and Control, pages pp. 2655-2660, December 1999.

[6] R. Langerak, J. W. Polderman, and G. T Krilavicius. Stability analysis for hybrid automata using conservative gains. IFAC Conference on Analysis and Design of Hybrid Systems ADHSO3, 2003.

[7] D. Liberzon. Switching in system and control. Systems and control : foundations and applications. Springer, 2003.

[8] D. Liberzon and A. Stephen Morse. Basic problems in stability and design of switched systems. IEEE Control Systems Magazine, 19(5):pp. 59-70, October 1999.

[9] S. Mitra and D. Liberzon. Stability of hybrid automata with average dwell time: an invariant approach. In Proceedings of the 43rd Conference on Decision and Control, pages pp. 1394-1399, December 2004.

[10] J. Tsitsiklis V. Blondel. Complexity of stability and controllability of elementary hybrid systems. Automatica, 35(3):pp. 479-489, 1999. 